\title{
Study protocol description: Dynamic Modelling of Resilience - Observational Study (DynaM-OBS)
}

Carolin Wackerhagen ${ }^{1, a}$, Ilya M. Veer, ${ }^{1,2, a}$, Judith M.C. van Leeuwen ${ }^{3, a}$, Sophie Bögemann ${ }^{3}$, Netali Mor ${ }^{4,5}$, Lara M.C. Puhlmann ${ }^{6,7}$, Zala Reppmann ${ }^{1}$, Antje Riepenhausen ${ }^{1,8}$, Aleksandra Uścitko ${ }^{9}$, Matthias Zerban ${ }^{10}$, Kenneth S.L. Yuen ${ }^{6,10}$, Göran Köber ${ }^{11,12}$, Shakoor Pooseh ${ }^{12}$, Jeroen Weermeijer ${ }^{13}$, Marta A. Marciniak ${ }^{14,15}$, Alejandro Arias-Vásquez ${ }^{3}$, Harald Binder ${ }^{11,12}$, Walter de Raedt $^{16}$, Birgit Kleim ${ }^{14,15}$, Inez Myin-Germeys ${ }^{13}$, Karin Roelofs ${ }^{3}$, Jens Timmer ${ }^{12,17,18}$, Oliver Tüscher $^{6,19}$, Talma Hendler ${ }^{4,5,20,21, b}$, Erno J. Hermans ${ }^{3, b}$, Raffael Kalisch ${ }^{6,10, b}$, Dorota Kobylińska ${ }^{9, b}$, Henrik Walter ${ }^{1,8, b}$

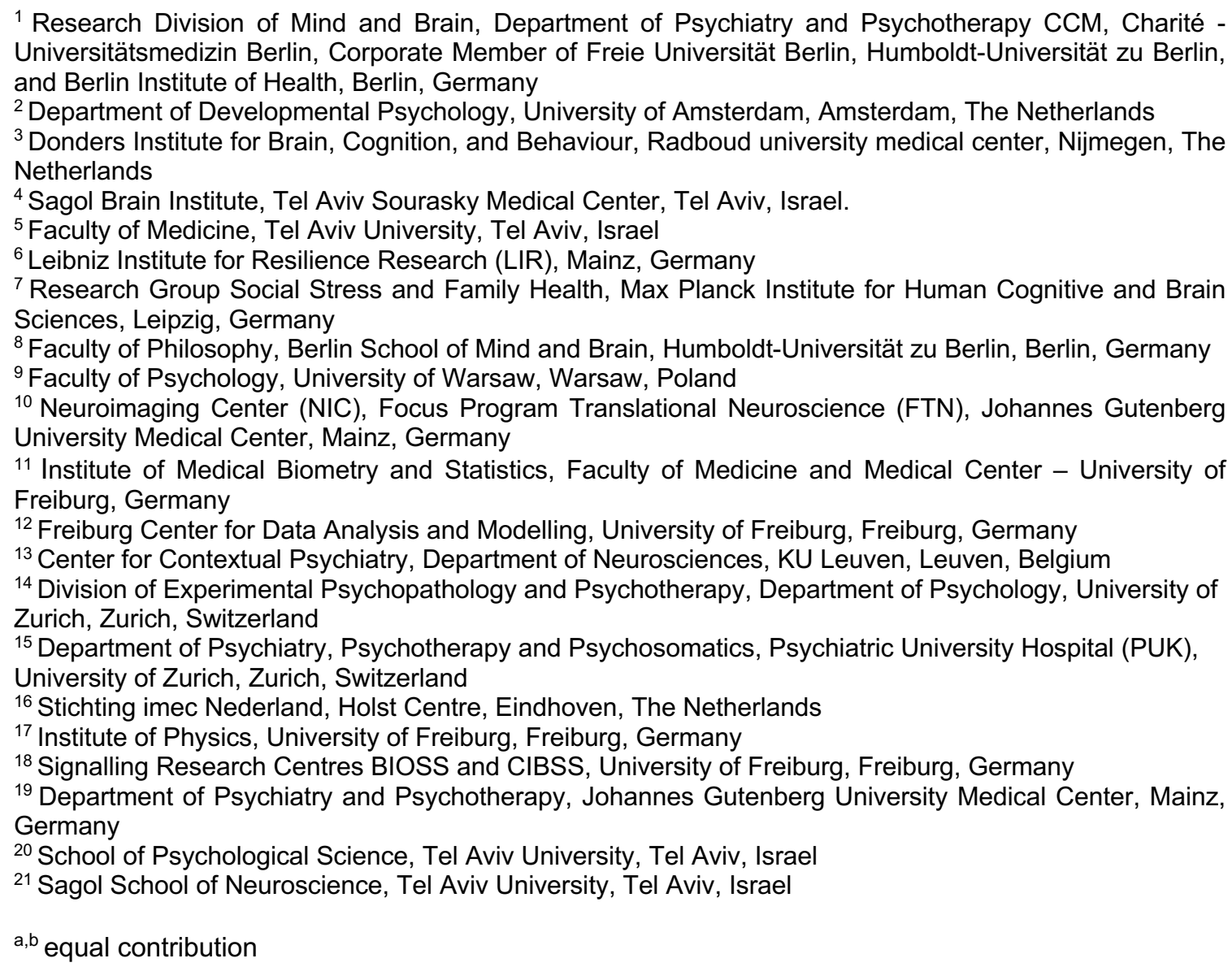

Corresponding author:

Dr. llya M. Veer

ORCID: 0000-0002-6733-3593

University of Amsterdam

Department of Developmental Psychology

Postbus 15916

1001 NK Amsterdam

The Netherlands

i.m.veer@uva.nl 


\section{Keywords}

Resilience; Stress; Stressor Reactivity; Resilience Factors; Mental Health; Stress Disorders; Longitudinal; Prospective; Neuroimaging; Ecological Momentary Assessment 


\begin{abstract}
Background

Stress-related mental disorders are highly prevalent and pose a significant burden on individuals and society. Improving strategies of their treatment and prevention requires knowledge about risk and resilience. This multi-center study aims to contribute to this endeavor by investigating psychological resilience in healthy, but vulnerable young adults over nine months. Resilience is operationalized as maintained or quickly recovered mental health despite exposure to stressors and assessed longitudinally in a frequent monitoring approach. We aim to investigate factors predicting, and adaptive processes and mechanisms contributing to mental resilience, and to provide a methodological and evidence-based framework for later intervention studies.
\end{abstract}

\title{
Methods
}

In a multi-center setting, across five research sites, a sample with the total target size of $\mathrm{N}=250$ male and female young adults is assessed longitudinally over nine months. Participants are included if they had an elevated level of (internalizing) mental health problems and reported at least three stressful life events while not affected by any mental disorder other than mild depression. At baseline, sociodemographic, psychological, neuropsychological, structural and functional brain imaging data, and salivary cortisol are acquired. In a six-months longitudinal phase I, bi-weekly online monitoring of stressor exposure, mental health problems, and positive appraisal style takes place, as well as monthly one-week ecological momentary assessments (EMA) and ecological physiological assessments (EPA). In a subsequent three-months longitudinal phase II, online monitoring is reduced to once a month and psychological resilience and risk factors are assessed again at the end of the nine-month period. In addition, genetic, epigenetic, and microbiome data are assessed at baseline, month three (microbiome only), and month six.

As an approximation of resilience, an individual stressor reactivity (SR) score will be calculated. Using regularized regression methods, network modeling, ordinary differential equations, landmarking methods, and neural net-based methods for imputation and dimension reduction, we will identify predictors and mechanisms of SR and thus be able to identify resilience factors and mechanisms that facilitate adaptation to stressors.

\section{Discussion}

The DynaM-OBS study provides a methodological framework and dataset to identify predictors and mechanisms of mental resilience, which are intended to serve as an empirical foundation for future intervention studies. 


\section{Background}

Stress-related disorders, such as depressive disorders, anxiety disorders, post-traumatic stress disorder, and addiction, are highly prevalent globally and pose a significant burden on individuals, the economy, and society in general [1-4]. Years lost to disability due to mental and substance use disorders have increased by 46.6 per cent from 1990 to 2016 [3], and mental disorders are associated with a reduced life expectancy with a median of 10.1 years [5]. Mental and substance use disorders are particularly prevalent in people in their twenties, and, although substance use and specific phobias tend to decrease in the thirties and forties, anxiety disorders and major depression have a high long-term stability [6]. Many studies in the field of mental health focus on mechanisms and predictors of vulnerability (risk) and dysfunction. However, we and others have argued that a focus on resilience, aiming to investigate factors and mechanisms contributing to the maintenance and recovery of mental health despite adversity [7], is a complimentary, helpful approach to reveal novel intervention targets and to help to avert (chronic) mental health problems before they develop. Here we describe the study protocol of such a longitudinal resilience study.

Resilience is the maintenance of or quick recovery towards mental health and well-being during and after times of adversity, such as trauma, difficult life circumstances, challenging life transitions, or physical illness $[8,9]$. It is becoming increasingly clear that resilience is the result of a dynamic process of successful adaptation to stressors [9-14]. Next to person-environment interactions and the activation of dispositional coping strategies, there is accumulating evidence that individuals change while they successfully cope with stressors. Hence, adaptation will frequently involve individual-level adjustments. These can manifest at various levels, such as altered perspectives on life [15], emergence of new strengths or competencies [16], partial immunization against the effects of future stressors [17-19], or as epigenetic alterations and modified gene expression patterns [20, 21]. Neurobiological studies in animal models indicate that adjustments at the level of the brain are causal for the preservation of normal behavior [2225]. In such a dynamic perspective, resilience itself is subject to change and not just inertia, insensitivity to stressors, or merely a passive response to adversity.

Consequentially, resilience should no longer be understood as a fixed personality trait or predisposition (the "resilient personality") that will determine successful coping independent of other factors. Rather, we should take a dynamic stance and investigate the complex, interactive, and time-varying processes ("resilience processes") that lead to a positive long-term outcome relative to the number of stressors an organism is exposed to. These processes will partly be determined by individual dispositions, including traits, habits and skills, beliefs, genotype, brain architecture, physical constitution, etc. ("resilience factors"). Although some of those will be quite stable and define a person's typical coping patterns throughout a stressful life period, other 
resilience factors may themselves undergo change, for instance by increasing their effectiveness or frequency of use. The latter would constitute allostatic resilience processes, in which the system learns to change its mode of operation in order to remain stable. Allostatic adjustment is more likely the more an external perturbation (a stressor) taxes the system. Homeostatic resilience processes, by contrast, are defined as mental stability in the absence of individual change [13].

By definition, resilience as an outcome cannot be measured through any one-time (crosssectional) assessment (e.g., a questionnaire, a brain scan, genotype, etc.) performed before adversity occurs, as the trait-like conceptualization of resilience implies. Instead, resilience can only be determined by assessing both stressors and mental health longitudinally, thus capturing the dynamic nature and time course of the stressors, as well as the changes in mental health that these stressors may or may not induce $[9,11]$. This should be complemented by assessing potential resilience factors at study baseline and, ideally, also repeatedly during the course of observation [13]. Moreover, the factors and processes of resilience should ideally be studied at different levels of organization, from the genetic/molecular, over physiological, neural, and cognitive up to behavioral, experiential, and social levels [26].

\section{The current study}

The observational study DynaM-OBS of the EU Horizon 2020 DynaMORE consortium ("Dynamic MOdelling of REsilience") was designed to unravel such multi-level mechanisms of outcome-based resilience. The targeted sample are young students finding themselves in the transition from family and school life into work or academics - a life period characterized by new unfamiliar environments and demands, which is associated in some individuals with the exacerbation of existing, or the onset of new, stress-related psychological problems. The rationale of focusing on young people is that many mental disorders have their first onset or even peak during this critical life transition phase [27] and stress-related mental problems appear to be a particular problem in the student population [28-33]. To enrich our sample with at-risk individuals, we added two further inclusion criteria: (i) participants must have a history of at least three adverse life events [34] and (ii) score in the mid-to-high range in the General Health Questionnaire (GHQ), a self-report instrument for internalizing symptoms [35], additionally to being a student or undergoing an apprenticeship. The study started in October 2020, when the second wave of the COVID-19 pandemic began in Europe, by which an additional general stressor was present in our sample population [36].

DynaM-OBS is a longitudinal study with a baseline assessment, a dense observation period of six months (longitudinal phase I) and a subsequent less dense observation period of three months (longitudinal phase II) in each participant. The DynaM-OBS study features four core 
components: (1) An extensive baseline characterization (baseline battery) measuring potential social, psychological, and biological resilience factors. (2) A bi-weekly online assessment of stressor exposure and mental health during phase I. (3) Repeated measurement of a subset of potential resilience factors at different time points during the study. (4) Every four weeks, oneweek-long ecological momentary assessments (EMA) and ecological physiological assessments (EPA) of mood and stress reaction patterns during real life are included using smartphones and wristbands, respectively.

DynaM-OBS' bi-weekly stressor and mental health assessments (core feature 2) apply the frequent stressor and mental health monitoring (FRESHMO) paradigm introduced recently to operationalize and measure resilience in longitudinal studies [13]. In the data analysis, we will use normative modeling of stressor reactivity as an approximation of resilience. Specifically, we will regress participants' average mental health problem score $P$ from all assessment time points in phase I over their average stressor exposure score $E$ from the same time points, and thus establish the sample's normative E-P relationship during the study. At any single assessment time point or series of subsequent time points, the deviation of a participant's (average) P score from the norm relationship (its regression residual) then expresses the participant's individual mental health reactivity to stressor exposure during that time window. A positive 'stressor reactivity' (SR) score reflects higher-than-predicted reactivity, whereas a negative SR score reflects lower-than-predicted reactivity. Importantly, SR scores inherently correct for different degrees of stressor exposure between individuals and can thus be compared between them.

On this basis, the inverse of the SR score calculated from average $E$ and $P$ scores over the entire phase I-time window of the study can be considered a dimensional measure of a participant's outcome-based resilience over these six months. We will also build withinparticipant time courses of SR scores, by building the score in sliding windows of several averaged bi-weekly $E$ and $P$ measurements. This permits us to index temporal fluctuations in reactivity and to detect potential substantial changes that would be a strong indicator of allostatic adjustment at the level of resilience factors, without which relevant increases or decreases in reactivity are hard to imagine.

Thus, the time series-based approach to stressor reactivity assessment goes beyond mere prediction of a longer-term resilience outcome (here: the inverted six-months SR score of phase I) from a single measurement of more or less stable resilience factors (here: DynaM-OBS baseline battery, core feature 2). In combination with a repeated measurement of resilience factors (DynaM-OBS core feature 3) - some bi-weekly, some at study entry and study exit - the approach allows for relating changes in the outcome to potentially underlying allostatic change in resilience factors (towards the good or the bad). Another, more distal source of change in the outcome may lie in the stressor exposure $(E)$ itself, which may rise to a degree that the system 
reacts allostatically, by ideally strengthening existing or developing new coping mechanisms, which then in turn improve stressor reactivity [13].

Core feature 4 of DynaM-OBS (EMA/EPA) serves to examine whether stressor exposure, mental health, and stressor reactivity measured with online questionnaires over periods of weeks or months (feature 2) are reflected in individuals' patterns stressor reactivity measured at a higher temporal frequency in real life. EMA combined with EPA will allow us to quantify, among others, frequency of self-reported real-life (micro)stressors, magnitude of mood or physiological changes in response to these, speed of recovery and alterations of recovery speed.

\section{Relation to other studies}

The FRESHMO paradigm in its combination with repeated resilience factor measurement is employed in several other longitudinal studies by our consortium and partners, including the "Mainz Resilience Project" (MARP; study protocol in preparation, see Kampa et al. [37, 38]), the "LOngitudinal Resilience Assessment" (LORA; [39]), and the "DynaMORE longitudinal study on psychological resilience to the mental health consequences of the Corona crisis" (DynaCORE-L; [40]). MARP and LORA are ongoing since 2016 and are monitoring stressors and mental health every three months and resilience factors approximately every 1.5 years over many years of participants' lives (see also [13]). DynaCORE-L was conducted over six weeks during the early phase of the COVID-19 pandemic in 2020 and involved weekly stressor, health, and factor monitoring. Like DynaM-OBS, all studies also include baseline resilience factor assessment. DynaM-OBS thus covers a time range lying in between MARP and LORA on the one hand, and DynaCORE-L on the other hand. A similar approach is also conducted in the Healthy Brain Study cohort study, which uses three waves of assessments to calculate dynamic resilience scores [41].

In DynaCORE-L, we observed that several baseline resilience factors prospectively predicted average SR over the full monitoring period, while week-to-week changes in resilience factors were not predictive of SR changes in the subsequent week (publication in preparation). This may indicate that allostatic adaptation processes may unfold on longer timescales. Another possibility is that the relatively mild stressor exposure in DynaCORE-L, which took place after the first wave of the pandemic had subsided, permitted homeostatic coping. With its longer time range and its more severe at-risk constellation, DynaM-OBS then should be more sensitive than DynaCORE-L in detecting allostatic processes. At the same time, DynaM-OBS allows us to test the generalizability of the baseline resilience factors discovered in DynaCORE-L to a different sample. Another source of hypotheses are yet unpublished preliminary analyses of the MARP and LORA data sets, most notably baseline predictions of the nine-months SR score there. In 
synopsis with the anticipated results from these long-term studies, DynaCORE-L and DynaMOBS will yield a good picture of the temporal dynamics of allostatic resilience processes.

DynaCORE-L had further detected that week-to-week changes in stressor exposure predate subsequent changes specifically in the resilience factor of active behavioral coping, which were, however, not translated into improvements in stressor reactivity. Again, this may have been the consequence of the limited duration or limited exposure severity in the study. With DynaM-OBS we therefore also aim to detect mediated relationships from stressors to resilience factors to stressor reactivity. The addition of EMA and EPA is intended to detect potential relationships at much higher (within-week and within-day) temporal resolution that is neither possible in DynaCORE-L nor in MARP and LORA.

Next to answering questions about resilience factors and processes, a purpose of DynaM-OBS is to establish a methodological framework for a subsequently planned intervention study (DynaM-INT) that aims to find new ways to prevent stress-related mental illness in at-risk individuals with the help of mobile training apps targeting specific resilience factors. In the context of such interventions, regular monitoring of stressor reactivity and resilience factors can provide information on the desired outcome (lastingly reduced SR) and potential working mechanisms (lastingly strengthened resilience factors), while a baseline battery of both resilience and risk factors may inform us about individual characteristics that predict intervention success.

\section{Statistical methods development}

The conceptual framework and the types of data generated by DynaM-OBS require new analysis methods. While the time-sensitive regression models employed in DynaCORE-L were appropriate for analyzing time point-to-time point (week-to-week) effects, they were also limited. Notably, they only tested each of the multiple, partially correlated resilience factors in a separate model and they did not consider interrelations between resilience factors; they did not test for time-lagged effects between either stressors and resilience factors or resilience factors and SR extending beyond one week; and they did not test for changes, or change points, in the time series of stressor exposure, resilience factors, or stressor reactivity. For this reason, we also use DynaM-OBS (together with the other mentioned studies) as a test bed for new methodological developments. These include regularized regression methods [36, 42], network models [12], landmarking methods [13], and individualized deep dynamic methods in combination with deep generative models allowing, e.g., additional quantifications of resilience, multiple imputation, and dimension reduction [42]. We will also use the data for potential improvements in the calculation of SR scores, including with partial least squares methods [43], serving notably to improve $P$ 
variance explanation by $E$ and better integration of stressors of various sources into $E$ [13]. Thus, DynaM-OBS has a strong exploratory character from a methodological perspective.

\section{Research questions and hypotheses}

We nevertheless formulate a set of hypotheses that will be tested with established methods, described in the Methods section. A major theoretical background of DynaM-OBS is Positive Appraisal Style Theory of Resilience (PASTOR) [9, 44]. Positive appraisal style (PAS) is the tendency to appraise potential threats to one's goals and needs (stressors) in a way that avoids unnecessarily negative but also highly unrealistically positive (delusional) appraisals. Instead, appraisals typically produced by individuals exhibiting PAS are in the range from realistic to slightly unrealistically positive. Thereby, positive appraisers generate appropriate, optimally regulated stress reactions, sufficient to cope with a threat but not using more resources than necessary and have more time for recovery and rebuilding of resources and more opportunity to make growth experiences than individuals that are inclined towards catastrophizing, pessimism, or helplessness.

\section{Questionnaires}

DynaM-OBS uses a PAS self-report questionnaire developed to assess the cognitive processes, or mental operations, that lead to positive appraisal contents (Positive Appraisal Style Scale process-based, PASSp; see Methods) in its baseline battery, its bi-weekly online monitoring, and in the exit questionnaire battery applied in month nine of the study. We hypothesize a) that baseline PASSp negatively predicts the SR score covering the first six months of the study (phase I, henceforth: $\mathrm{SR}_{\mathrm{I}}$ ), b) that changes in PASSp from time point to time point in the biweekly monitoring inversely co-fluctuate with the corresponding SR score, $\mathrm{c}$ ) that such changes also negatively predict time-lagged changes in SR (where the duration of PASSp change necessary to entail SR changes and the duration of the time lag are to be explored), and d) that changes in PASSp from study baseline to study exit are negatively related to the SR score covering the entire study period (phases I and II, SR ${ }_{I+I I}$ ).

An alternative PAS self-report instrument used in the DynaM-OBS baseline and exit batteries focuses on positive appraisal contents instead of focusing on the processes that generate such appraisals (PASS - content-based, PASSc; see Methods). Above hypotheses a) and d) therefore analogously apply to PASSc.

We will also test in this fashion other baseline and exit questionnaires that specifically assess positive appraisal tendencies on single threat appraisal dimensions, namely the dimensions of threat probability (optimism), threat coping potential (general self-efficacy and control), and threat magnitude/costs (inverted anxiety sensitivity score). In combined multi-variate analysis 
using regularized regression of the PAS scales and the latter instruments, we will also address the question of which of these scores are best in explaining SR.

Finally, we will ask whether PAS (assessed with the 'winning' instrument or instruments in the comparative multi-variate analysis) mediates the effects of perceived social support on SR and whether its effect on SR is in turn mediated by perceived good stress recovery (see $[9,36]$ for rationale). These mediation analyses will again use either baseline scores (for explanation of $\mathrm{SR}_{\mathrm{l}}$; a) or baseline-to-exit change scores (for explanation of $\mathrm{SR}_{1+1 \mid}$; d).

The complete battery of resilience and risk factors assessed in baseline and exit questionnaires will be evaluated for their capacity to explain SR scores in a more exploratory fashion, using above and potential newly developed methodological approaches.

\section{Neuroimaging}

In the development of PASTOR, we have emphasized the problems associated with self-report assessment in general (which knows various sources of bias) and self-report assessment of appraisal processes and contents in particular (not all of which may be accessible to consciousness and verbally reportable) [9]. We therefore here also use a complementary approach, namely, to indirectly index the effectiveness and/or efficiency of participants' positive appraisal processes through objective measures of their behavioral, physiological, or neural reactions to stressors in the lab or in real life. To this purpose, we generated a baseline neuroimaging battery where functional magnetic resonance imaging (fMRI) tasks are (partly) accompanied by behavioral and physiological recordings. The battery includes tasks from the corresponding MARP battery (reward sensitivity, differential fear conditioning, and situationfocused volitional reappraisal [37], all of which provide reliable imaging data [38] and were either found to be predictive of SR in initial analyses in MARP (unpublished) or else were retained because of their special theoretical interest (see [37] for task rationales in the context of PASTOR). A purely behavioral stress reactivity and recovery task in the MARP battery is replaced in the DynaM-OBS battery by a dedicated neuroimaging variant $[45,46]$. To link the battery with other neuroimaging cohort studies, a frequently used implicit emotional processing task is also employed $[47,48]$. These tasks are complemented by structural MRI and restingstate $\mathrm{fMRI}$ measurements.

Like the longitudinal resilience data, the neuroimaging data will also be used for analysis methods development. Specifically, we hypothesize (based on initial MARP findings) that SR will be negatively predicted by activity related to a) high gain anticipation (gain>zero anticipation contrast), b) low loss anticipation (loss>zero anticipation contrast), c) high threat-safety discrimination (CS+>CS- contrast), d) low threat generalization (CS- contrast), e) high volitional 
reappraisal ( $R>N R$ contrast), and $f$ ) high amygdala-ventromedial prefrontal functional connectivity during faces processing [49]. For details on contrasts, see [37].

\section{Other data modalities and hypotheses.}

PASTOR claims that the effects of other social, psychological, and biological resilience factors on resilience are mediated by how they shape PAS [9]. In this framework, we use a range of additional questionnaires, neuropsychological tests, and bio-samples (blood for genotyping, DNA-methylation, plasma proteome analysis and at one site also cytokines, stool for gut microbiome analysis, and saliva for cortisol analysis). Socio-demographic variables and wellknown risk factors are also assessed. 


\section{Methods/Design}

\section{Study centers and study period}

The data was acquired in a multi-center setting at five research sites: Charite -

Universitätsmedizin Berlin, Department of Psychiatry and Psychotherapy in Berlin, Germany; Universitätsmedizin Mainz, Neuroimaging Center (NIC) in Mainz, Germany; Donders Centre for Cognitive Neuroimaging (DCCN) in Nijmegen, The Netherlands; Sagol Brain Institute, Tel Aviv University (TAU) and Tel Aviv Soursaky Medical Center, Tel Aviv, Israel; University of Warsaw, Faculty of Psychology in Warsaw, Poland. Data acquisition started in October 2020, a period during which the second wave of the COVID pandemic was after or at its peak at most sites. Completion of the baseline assessments was in August 2021, completion of phase I assessments is expected in February 2022, completion of phase II assessments in May 2022.

\section{Participants}

Two-hundred-fifty mentally healthy male or female participants who were studying or in vocational training at the time of recruitment were planned to be included at the five research sites ( $\mathrm{N}=50$ each). The exact number of participants per site will be reported after completion of data acquisition. The age range was 18-25 at all sites, except for TAU, where the age range was 18-27 because most young adults in Israel complete 2-3 years of military service and spend one year abroad before entering vocational training or university. Participants were included if they had experienced three or more stressful life events [34], which they rated as burdening, and if they reported an elevated level of general psychopathology (internalizing symptoms) as defined by a score of $>20$ in the General Health Questionnaire (GHQ-28) [35]. The complete list of inclusion criteria is provided in Table 1.

\section{Materials}

\section{Self-report variables}

Self-report variables contained demographic characteristics, stressor exposure, mental health, as well as potential psychological resilience and - to a lesser extent - risk factors. Resilience and risk factors (RFs) are grouped into primary and secondary RFs. Primary RFs are of main interest in the current study based on previous findings and theoretical background of our consortium [9, 36, 40], while secondary RFs are based on hypotheses drawn from the literature. Where available, validated versions of questionnaires and their translations into the site-specific languages were used. Self-developed questionnaires are provided at https://osf.io/qj9mf/. See Table 4 for an overview of all questionnaires. 


\section{Neuropsychological measures}

The neuropsychological test battery included paper-pencil paradigms such as the Trail Making Test $[50,51]$, to assess visual attention and task switching speed, the HAWIE Matrices, to assess non-verbal logical reasoning [52], and the HAWIE Digit Symbol Test, to assess processing speed [52]. Further, a computer-based paradigm, the Stab/Flex task [53, 54] was administered to assess cognitive flexibility (see section 1 and Figure $S 1$ of the supplementary information).

\section{Neuroimaging}

\section{MRI data acquisition}

At all sites except for Warsaw, brain imaging data were acquired on identical models of 3T MAGNETOM Prisma systems (Siemens Healthineers, Erlangen, Germany) with 32-channel head coils (at TAU 64-channel head coil) using the following settings: Multiband gradient-echo echo planar imaging $(\mathrm{EPI})$ sequences $\left(\mathrm{TR}=800 \mathrm{~ms}, \mathrm{TE}=37 \mathrm{~ms}\right.$, flip angle $=52^{\circ}, \mathrm{FOV}=208$ $\mathrm{mm}$, voxel size $=2.0 \times 2.0 \times 2.0 \mathrm{~mm}, 72$ slices, $\mathrm{MB}$ acceleration factor $=8$, phase-encoding direction = PA) from the Center for Magnetic Resonance Research, University of Minnesota (CMRR), as adopted from the Human Connectome Project, were used for blood oxygen-level dependent (BOLD) fMRI [55]. Before each task, a pair of blip-up/blip-down EPI sequences was acquired $\left(\mathrm{TR}=8000 \mathrm{~ms}, \mathrm{TE}=66 \mathrm{~ms}\right.$, flip angle $=90^{\circ}, \mathrm{FOV}=208 \mathrm{~mm}$, voxel size $=2.0 \times 2.0 \mathrm{x}$ $2.0 \mathrm{~mm}$ ), one with an AP and one with a PA phase-encoding direction. Further, a T1-MPRAGEsequence $\left(\mathrm{TR}=2500 \mathrm{~ms}, \mathrm{TE}=2.22 \mathrm{~ms}\right.$, flip angle $=8^{\circ}, \mathrm{FOV}=256 \mathrm{~mm}$, voxel size $=0.8 \times 0.8$ $x 0.8 \mathrm{~mm}$ ), a FLAIR sequence $\left(\mathrm{TR}=9000 \mathrm{~ms}, \mathrm{TE}=83 \mathrm{~ms}\right.$, flip angle $=150^{\circ}, \mathrm{FOV}=220 \mathrm{~mm}$, voxel size $=0.7 \times 0.7 \times 3.0 \mathrm{~mm}$ ), and two diffusion-weighted imaging sequences (TR $=3600 \mathrm{~ms}$, $\mathrm{TE}=92 \mathrm{~ms}$, flip angle $=78^{\circ}, \mathrm{FOV}=210 \mathrm{~mm}$, voxel size $=2.0 \times 2.0 \times 2.0 \mathrm{~mm}, 50$ directions $\mathrm{b}=$ 1000,50 directions $b=2000,5$ b0 volumes, $M B$ acceleration factor $=3$, phase-encoding direction $=\mathrm{AP})$ and $\left(\mathrm{TR}=3600 \mathrm{~ms}, \mathrm{TE}=92 \mathrm{~ms}\right.$, flip angle $=78^{\circ}, \mathrm{FOV}=210 \mathrm{~mm}$, voxel size $=$ $2.0 \times 2.0 \times 2.0 \mathrm{~mm}, 6$ directions $\mathrm{b}=2000$, MB acceleration factor $=3$, phase-encoding direction = PA), adopted from the UK Biobank scan protocol [56] were acquired.

In Warsaw, a 3T MAGNETO Trio system (Siemens, Germany) was used. There, multiband gradient-echo EPI sequences were acquired with the following settings: $\mathrm{TR}=1410 \mathrm{~ms}$, TE = $30.4 \mathrm{~ms}$, flip angle $=56^{\circ}, \mathrm{FOV}=210 \mathrm{~mm}$, voxel size $=2.5 \times 2.5 \times 2.5 \mathrm{~mm}, 60$ slices, MB acceleration factor $=3$, phase-encoding direction $=$ PA. Blip-up/blip-down EPI sequences before each task (identical settings as other sites, except for voxel size $=2.5 \times 2.5 \times 2.5 \mathrm{~mm}$ ), T1MPRAGE $\left(\mathrm{TR}=1100 \mathrm{~ms}, \mathrm{TE}=3.32 \mathrm{~ms}\right.$, flip angle $=7^{\circ}, \mathrm{FOV}=256 \mathrm{~mm}$, voxel size $=1.0 \times 1.0$ $x 1.0 \mathrm{~mm}$ ), a FLAIR sequence with identical settings as above, and two diffusion-weighted imaging sequences (identical settings as above, except TE $=97 \mathrm{~ms}$ ) were acquired as well. 
Head movement was restricted by foam pads and a tape on the forehead. All task paradigms were presented using the software Presentation $®$ (Neurobehavioral systems, www.neurobs.com on a monitor placed behind the scanner bore via a mirror that was fixed on the head coil.

\section{Reward sensitivity}

To capture neural responses during reward and loss anticipation, an adapted version of the monetary incentive delay task (MID) [57] was used, described in detail by Kampa and colleagues [37]. Participants were instructed that they can win or lose a small amount of money if they pressed a button fast enough as soon as the target stimulus appeared on the screen. Before the target appeared, participants were presented with a cue for 2 seconds, which indicated if they could win or lose money on the current trial $(+3 € / 12 \mathrm{NIS} / 12 \mathrm{PLN}$, $+0.5 € / 2 \mathrm{NIS} / 4 \mathrm{PLN}, \pm 0,-0.5 € / 2 \mathrm{NIS} / 4 \mathrm{PLN},-3 € / 12 \mathrm{NIS} / 12 \mathrm{PLN})$. The cue was followed by a jittered anticipation phase of 2-2.5 s, after which participants had to press a button as soon as a target stimulus (white star) appeared on the screen. Each trial ended with a $2 \mathrm{~s}$ numeric feedback on subjects' trial outcome and the overall gain. To assure that the experience of reward did not differ between subjects depending on task performance, an adaptive algorithm was applied that changed the target duration for the participant within each condition based on their past performance. If the participant's hit rate was below $66 \%$, the target duration was increased by 25 $\mathrm{ms}$; else, it is reduced by $25 \mathrm{~ms}$. Reaction times and hit rates were collected as behavioral outcomes. A graphical depiction of the task design is provided in supplementary Figure S2.

\section{Differential fear conditioning}

We used the fear conditioning part of a safety learning and memory paradigm, described by Kampa et al. [37]. Three different geometrical shapes (square, circle, and triangle) served as conditioned stimuli (CS). Electro-tactile stimuli delivered to the back of the right hand were used as unconditioned stimuli (US). Two background images of different conference rooms were inherited from the original paradigm in which they served as context variable. They each showed a screen on which the CSs were depicted to make them part of the scene in a naturalistic way (see supplementary Figure S3). Before the main experiment, a short training was given during which the different stimuli and the rating scale were shown, but no electric stimuli were delivered. During the experiment, two of the CSs were paired with a US in $100 \%$ of the trials. These stimuli worked as CS+. The other stimulus (CS-) was never paired with a US. Each CS trial lasted $6 \mathrm{~s}$. During the first $4.5 \mathrm{~s}$ of a trial, participants rated their fear of receiving an electric stimulus between 1 and 100 using a visual analog scale at the bottom of the screen. Inter-trialintervals lasted 9 to $15 \mathrm{~s}$. Background images and stimuli were counterbalanced across participants. 
The stimulus intensity was calibrated beforehand using a calibration procedure developed to reach a stimulus level that was highly unpleasant, but not painful. Participants received an initial stimulus at the lowest level (1) and were asked to rate its severity on a scale from $1=$ "not unpleasant at all" to $5=$ "painful". The desired rating level was 4 . The stimulus intensity was adapted after each stimulus according to a predefined scheme (supplementary Figure S4). Stimulation was given through two sticky electrodes (INVISATRACE Adult ECG Electrode) on the back of the hand. Except for the DCCN, all sites used a Digitimer DS7A stimulator (Welwyn Garden City, UK). One electrical stimulation consisted of a train of three square-wave pulses of $2 \mathrm{~ms}$ duration each, with an interval of $50 \mathrm{~ms}$ apart from each other. Electric potential was $400 \mathrm{~V}$ and current varied with calibration, starting at a minimum of $10 \mathrm{~mA}$. At the DCCN, the electric stimuli were delivered using an Innostim Tens 2000 (Formerly named MAXTENS2000, BioProtech Inc, Gangwon-do, Korea) [58]. Stimulus duration was $200 \mathrm{~ms}$, and intensity varied between $0 \mathrm{~V}-40 \mathrm{~V} / 0 \mathrm{~mA}-80 \mathrm{~mA}$.

At TAU, the electric stimulus was replaced by a white noise with a duration of $50 \mathrm{~ms}$, volume turned to max to create a startle response. All other conditions (background, reinforcement scheme) remained the same. A pilot study at TAU revealed similar activations as the typical activation patterns found using an electric stimulus.

\section{Situation-focused volitional reappraisal}

In this task, participants were instructed to positively reinterpret or just view photographs which are either negative, positive, or neutral and to rate their affective state on a non-verbal scale. The paradigm has been previously described by Kampa and colleagues [37] and was adapted from Kanske and colleagues [59]. Stimuli were selected from the International Affective Picture System (IAPS) and EmoPics [60] based on normative ratings in valence and arousal [61]. In a fully balanced, three-by-two factorial design, the three types of picture valences were combined with either situation-focused reappraisal or viewing the pictures as a control condition (see supplementary Figure S5).

\section{Implicit emotion processing}

To assess neural responses during implicit emotion processing, we used an adaptation of the face matching task [47, 48]. In each trial, participants were presented with a trio of pictures and were instructed to select the matching pair by pressing a button. In the emotion condition, the trios contained gray scale photographs of Ekman faces [62] with angry or fearful expressions, counterbalanced for sex and emotion valence. In the control condition, the trios contained geometric shapes (circles, horizontal ellipses, and vertical ellipses). Four blocks per condition 
were presented in alternation. Each block consisted of one instruction (2s) and 6 trials (5s each), see supplementary Figure S6 for the task design.

\section{Resting state}

A resting-state scan was acquired before and after the stress task, during which participants were instructed to keep their eyes open and focus on a fixation cross on the screen.

\section{Social stress}

To examine brain activation and cortisol levels in response to stress, an adaptation of the ScanSTRESS paradigm was employed [63-65]. In this task, participants were instructed to perform mental rotation and arithmetic subtraction exercises. During the performance, task speed and difficulty was automatically adjusted so that participants fail most of the tasks. Further, participants were presented with a live video screen showing the face of the experimenter observing and giving negative non-verbal feedback on the performance. The task thus involves both social evaluative threat components (verbal and non-verbal feedback by the experimenter), and uncontrollable components (task difficulty, time constraints, and mock feedback of poor performance). The original version of the task is composed of two runs, both containing control (no feedback, no video) and stress (feedback and live video of experimenters) blocks, one run before and one after negative verbal feedback by the experimenter. Here we used the adapted version by Sandner and colleagues [46], in which all stress blocks are presented in one run and all control blocks in another run, both presented before and after negative verbal feedback by the experimenter. However, we shortened this version by discarding the control runs and using the stress runs only. Specifically, a shorter practice run of stress blocks was first presented, following by negative verbal feedback, after which a full run of stress blocks was presented during scanning. Brain responses to the stress paradigm are estimated from the comparison of task blocks vs. baseline (fixation cross). An overview of the task design is provided in supplementary Figure S7.

\section{$\underline{\text { Ambulatory assessments }}$}

During monthly burst weeks (six days) ecological momentary assessments (EMA) and ecological physiological assessments (EPA) were measured.

\section{$E M A$}

Each burst week, participants received a smartphone (Motorola Moto E6 Play) with the RADAR active RMT application for EMA data collection (https://radar-base.org/). Questionnaires were sent at ten time points ("beeps") per day during the participants' usual wakening hours using push notifications. Notifications were therein scheduled to appear within 90-minute blocks, semi- 
randomly; that is, for all participants the notifications appeared at the same time. Similarly, two notifications never occurred within 15 minutes of each other (see supplementary Table S1 for the beep schedule). Participants received a reminder notification 5 minutes after the initial notification and the EMA questionnaire remained available for 10 minutes after the initial push notification. Every beep questionnaire ( $\sim 3 \mathrm{~min})$ included in-the-moment self-assessments of mood, (virtual) social context, physical context, event appraisal, substance use, and anticipation of pleasure. Additionally, participants were instructed to start a separate morning questionnaire ( 1 min) about the last night's sleep immediately after waking up. Right before going to bed, participants were instructed to start an evening questionnaire ( $\sim \mathrm{min})$ about the evaluation of the day, the most negative and positive event of the day, stress anticipation for the upcoming day and whether the questionnaire influenced their mood during that day. All EMA items are provided in supplementary Figures S8 and S9.

\section{$E P A$}

EPA was measured for 23 hours a day during each burst week using the Chill+ wristband developed by IMEC (https://www.imec-int.com/en/chill). The wristband measures photoplethysmograph-based heart rate, galvanic skin response, skin temperature, and movement through a 3-axis accelerometer and 3-axis gyroscope. Further, participants were instructed to press a button on the wristband to actively report stressful events.

\section{Procedure}

Participants underwent a screening for inclusion criteria, two assessment days at baseline (Table 2), and longitudinal follow-up assessments (phase I and phase II, see Table 3). Before each on-site appointment, a short screening interview about potential COVID-19 symptoms including measurements of or questions regarding body temperature were conducted, to minimize risk of transmission. Participants and experimenters wore FFP2 or surgical masks, used disinfectants, and kept a distance of at least 1.5 meters.

\section{$\underline{\text { Recruitment and pre-screening }}$}

Participants were recruited via online advertisements in mailing lists and on social media platforms. After receiving brief information about the study purpose, methods and prerequisites, participants were invited to anonymously fill out an online screening survey via the platform SoSci Survey (www.soscisurvey.de), in which eligibility criteria (Table 1) of the study were checked using an automated algorithm. When starting the screening survey, participants were instructed to generate an anonymous code, which they later provided to the study staff in case of their inclusion to link their screening data to their participant ID. Screening data of nonincluded participants remained anonymous. After filling out the online screening questionnaire, 
eligible participants received an invitation to participate and a request to contact their study site. The complete study information material was then sent to the participant via email and an on-site appointment was made.

\section{On-site screening}

During the on-site appointment, participants received verbal information about the study and gave their written informed consent. Then, further inclusion criteria were assessed in a standardized interview with trained researchers (MINI, Sheehan et al., 1998, Table 1). Afterwards, a urine-based drug screen test (SureStep ${ }^{\mathrm{TM}}$ Multi-Drug One Step Screen Test Panel, Innovacon Inc., USA) for amphetamine, barbiturates, benzodiazepines, buprenorphine, clonazepam, cocaine, fentanyl, heroin, ketamine, cannabis, methadone, methamphetamine, methylenedioxymethamphetamine, morphine, opiate oxycodone, phencyclidine, propoxyphene, tramadol and tricyclic antidepressants was administered, after which participants who were included started with the baseline assessment (baseline day 1).

\section{Baseline day 1}

Baseline assessments were distributed across two appointments on two days (Table 1).

\section{Neuropsychological assessments}

During neuropsychological assessments, one participant per session was assessed by one study assistant. The participant was placed at a desk with a computer in a room with minimized potentially distracting stimulation (e.g., noise, visual distractions, other people). Telephones were muted or in airplane mode. The first task (Stab/Flex) was presented on the computer, all other tasks were instructed verbally and executed in paper-pencil format (see Materials section).

\section{Blood sampling}

One blood sample was collected at baseline day 1 or baseline day 2 and one at month 6 (see Table 3). Nine $\mathrm{mL}$ of blood (10 at DCCN) were drawn from each participant into an EDTA tube (red monovette; Sarstedt, Nümbrecht, Germany) and stored as whole-blood at $-20^{\circ} \mathrm{C}$ or colder until assay of DNA and DNA-methylation. At the study sites NIC and DCCN an additional $9 \mathrm{~mL}$ (NIC) or $10 \mathrm{~mL}$ (DCCN) of blood were sampled into EDTA tubes for proteomic analyses. At these sites, all blood was drawn between 12:30 and 15:30 and participants arrived at least 5 hours sober to limit the influence of metabolism or diurnal oscillations on proteomics measurements. Blood samples for proteomics assay were centrifuged and serum was divided into 8-16 aliquots (depending on volume), which were stored at $-80^{\circ} \mathrm{C}$ until assay. At TAU, two additional tubes (one EDTA, one VACUETTE® TUBE $3.5 \mathrm{ml}$ CAT Serum Separator Clot 
Activator) of blood samples were taken between month 1 and month 6 to derive IL-10, IL-6, TNFa and CRP.

\section{Stool sampling}

Stool samples were collected using an OMNIgene-gut feces kit (OM-200, DNAgenotek). Participants received a test kit, an instruction sheet about the collection procedure, the Bristol Stool Scale [67], and a verbal instruction by the test leader. Participants were instructed to collect the stool sample as close as possible to the appointment, to take several small samples from different locations in the stool material, to fill out the Bristol Stool Scale, and to store the sample at a dark place without direct sunlight. Participants brought the sample to the study center at the next appointment, where it was stored at $-20^{\circ} \mathrm{C}$ until assay of gut microbiome, or, at $\mathrm{DCCN}$, directly shipped it to the laboratory processing the microbiome).

\section{Post-assessment procedures}

After the baseline day 1 assessments, a personalized schedule was created with the participant, indicating the upcoming MRI appointment and timings of the upcoming phase I and phase II assessments. Further, participants received all necessary information about the online questionnaires and were introduced into the online survey platform SoSci Survey (www.soscisurvey.de) by filling out a dummy questionnaire. Finally, to ensure the participant's well-being, they were asked if they have experienced emotional disturbances triggered by any questions asked during the preceding session in a standardized interview. In case they reported emotional disturbance and a need for help, they were directed to a clinician associated with the study.

\section{Baseline day 2}

At baseline day 2, neuroimaging and a briefing about the ambulatory assessments took place. Before testing, participants returned their stool sample (except for DCCN), and another urinebased drug screen test (see description at baseline day 1 ) was conducted. Participants with a negative test result then received a brief training session of the MRI paradigms. During the training, they were presented with an on-screen presentation of the tasks while the test leader explained the tasks and asked questions to ensure the participant had understood the instructions. To account for diurnal or metabolism-related variation in cortisol levels, all scanning took place between 13:00 and 17:00 hours in the afternoon. Participants were instructed to get up from bed at least four hours before the appointment, not to eat, smoke, or drink beverages containing caffeine or sugar at least two hours before starting the MRI, to refrain from doing physical exercises on that day, and not to drink alcohol within 24 hours before the appointment. They were reminded of that via email prior to the appointment. 
Physiological and subjective measures during neuroimaging

During all fMRI sequences, the participants' heart rate was assessed with a wireless pulse oximeter (Siemens Healthineers, Erlangen, Germany). Further, to assess salivary cortisol levels in response to the MRI tasks, nine saliva samples were collected before, in between, and after the scanning sequences using Salivette collection kits (Sarstedt, Nümbrecht, Germany). With each saliva sample, the participant's subjective level of distress was assessed on a scale from 0 $=$ not stressed to $10=$ extremely stressed. Before scanning, participants were introduced into the saliva sampling technique while donating their first saliva sample. The participant received a plastic tube containing a cotton swab and was instructed to put the cotton swab into their mouth without touching it with their fingers. They were instructed to chew and moisten the cotton swab for one minute and to put it back into the plastic tube. This procedure was repeated for eight times, of which the participant was inside the scanner five times (see Table 1).

\section{Neuroimaging}

When placed in the MRI scanner, participants were provided with earplugs. To their right hand, they received a 4-button Inline Fiber Optic Response Pad (Current Design, www.curdes.com; home-designed system in Warsaw) and an electrode for the fear conditioning task was attached on the back of the right hand. The wireless pulse oximeter was attached to the index finger of their left hand. Via a mirror placed on the head coil, they were presented with the visual stimulation of the tasks on a monitor placed behind the scanner bore. Before and after each task, the test leader gave verbal instructions via an intercom system and received feedback from the participant. The specific instructions were repeated verbally on screen and by the test leader before each task. An overview of the procedure steps during neuroimaging and further details of the tasks are provided in supplemental information. After scanning, participants were asked to fill out an MRI exit interview questionnaire (paper-pencil), which asked about experiences and potential difficulties with the fMRI tasks.

\section{EMA / EPA briefing}

After scanning, participants were thoroughly briefed about the EMA and EPA devices and procedures. Contraindications for using the wristband collecting EPA (skin disease around the wrist area, wounds or skin allergies, medication with phototoxic side effects) were ruled out. The devices (mobile phone, wristband, and chargers) were handed to the participants and their functions were explained. Further, the purpose of EMA/EPA, the app, and questionnaires were explained, and it was discussed with the participant how to ensure that beeps are not missed in every-day life. Further details are described in the materials section and supplemental information. 


\section{Post-MRI debriefing}

After donating their last saliva sample, participants were debriefed about the stress task (see materials section) and got informed that the task was programmed to adapt to the participant's performance and aimed at inducing stress, instead of measuring their cognitive performance. Further, identical to baseline day 1 , participants were interviewed about emotional disturbances triggered by any questions asked during the preceding session in a standardized fashion. In case they reported emotional disturbance and a need for help, they were offered counseling by a clinician associated with the study.

\section{Longitudinal Phase I}

In the first weeks after baseline day, a baseline battery of questionnaires was assessed online. Longitudinal phase I spanned from month 1 to month 6 and contained the online monitoring for the FRESHMO paradigm [13]: In bi-weekly online questionnaires, stressor exposure (E), mental health problems $(\mathrm{P})$, and process-based positive appraisal style (PASSp) were assessed online. For this, participants received a link to the SoSci Survey platform at the beginning of each online monitoring week and had one week to fill out the questionnaire. Further, one-week EMA/EPA assessments took place at the second week of each of the six study months, for which participants received 10 beeps per day (see Table 3),

Before and after the EMA/EPA weeks, participants came to the lab to pick up / return the devices used for EMA/EPA (smartphones and wristbands). During these appointments, participants were again interviewed about potential emotional disturbances to ensure their wellbeing. In month 3 and month 6 , further stool samples were taken. In month 6 , a second sample of blood was taken (see Table 3).

\section{Longitudinal Phase II}

In longitudinal phase II, which spanned from month 7 to 9 , assessments of $E, P$, and PAS took place only once a month and no EMA/EPA was conducted. The final questionnaire battery at month 9 contained measures of additional resilience factors (see Table 3).

\section{$\underline{\text { Remuneration }}$}

Complete participation in all assessments was remunerated with 290 EUR (in Tel Aviv 1224 NIS, in Warsaw 1200 PLN). Further, participants could win about 10 EUR on average during the reward task in the neuroimaging battery. Furthermore, those who finished all assessments until month six, week three, were included in a lottery to win one out of three additional 100 EUR / 400 NIS / 400 PLN. To maintain compliance throughout the longitudinal assessments, money was disbursed in tranches at different timepoints throughout the study, see Supplementary Table S2. 


\section{Discussion}

The DynaM-OBS study targets a gap in resilience research: providing a dense longitudinal database to assess resilience as a long-term outcome and underlying resilience processes. This approach and framework offer new possibilities to test current hypotheses about putative resilience factors as well as to explore novel ones. The results and database generated in DynaM-OBS have the potential to serve as a foundation for future studies on interventions and trainings, aiming to enhance resilience factors and adaptive processes in at-risk individuals. 


\section{List of abbreviations}

\begin{tabular}{|c|c|}
\hline 3T & 3 Tesla \\
\hline AP & Anterior-to-posterior \\
\hline BOLD & Blood oxygen-level dependent \\
\hline CMRR & Center for Magnetic Resonance Research, University of Minnesota \\
\hline COVID-19 & Coronavirus disease 2019 \\
\hline CRP & C-reactive protein \\
\hline CS & Conditioned stimulus \\
\hline DCCN & Donders Centre for Cognitive Neuroimaging \\
\hline DynaCORE-L & $\begin{array}{l}\text { DynaMORE longitudinal study on psychological resilience to the mental health } \\
\text { consequences of the Corona crisis }\end{array}$ \\
\hline DynaM-INT & DynaMORE Intervention Study \\
\hline DynaM-OBS & DynaMORE Observational Study \\
\hline DynaMORE & Dynamic MOdelling of REsilience \\
\hline $\mathrm{E}$ & Stressor exposure \\
\hline EDTA & Ethylenediamine tetraacetic ac \\
\hline EMA & Ecological momentary assessments \\
\hline EPA & Ecological physiological assessments \\
\hline EPI & Echo planar imaging \\
\hline EUR & Euro \\
\hline FLAIR & Fluid-attenuated inversion recovery \\
\hline fMRI & Functional magnetic resonance imaging \\
\hline FOV & Field of view \\
\hline FRESHMO & Frequent stressor and mental health monitoring \\
\hline GHQ & General Health Questionnaire \\
\hline IAPS & International Affective Picture System \\
\hline IL & Interleukin \\
\hline IMEC & Interuniversity Microelectronics Centre \\
\hline LORA & LOngitudinal Resilience Assessment \\
\hline MARP & Mainz Resilience Project \\
\hline MB & Multiband \\
\hline MID & Monetary incentive delay \\
\hline MINI & Mini-international neuropsychiatric interview \\
\hline $\mathrm{mL}$ & Milliliters \\
\hline $\mathrm{mm}$ & Millimeters \\
\hline ms & milliseconds \\
\hline NIC & Neuroimaging Center Mainz \\
\hline NIS & New Israeli Shekel \\
\hline $\mathrm{P}$ & Mental health problems \\
\hline PA & Posterior-to-anterior \\
\hline PAS & Positive appraisal style \\
\hline PASSp & Positive Appraisal Style Scale - process-based \\
\hline PASTOR & Positive Appraisal Style Theory of Resilience \\
\hline PCA & Principle component analysis \\
\hline PLN & Złoty \\
\hline pRMT & Passive remote monitoring technology \\
\hline RF & Resilience and risk factors \\
\hline S & Seconds \\
\hline SR & Stressor reactivity \\
\hline TAU & Tel Aviv University \\
\hline TE & Time to echo \\
\hline TNF-a & Tumor necrosis factor a \\
\hline TR & Repetition time \\
\hline UK & United Kingdom \\
\hline US & Unconditioned stimulus \\
\hline
\end{tabular}




\section{Declarations}

\section{Ethics approval and consent to participate}

The study was reviewed and approved by the local ethics committees of all participating sites: The ethics committee of Charité - Universitätsmedizin Berlin, Germany; the ethics committee of Radboud University Medical Center, Nijmegen, The Netherlands (CMO Arnhem-Nijmegen); the ethics committee of the Tel Aviv University, Tel Aviv, Israel and the Helsinki committee of Tel Aviv Souraski Medical Center; the ethics committee of the State Medical Board of RhinelandPalatinate, Mainz, Germany; the Ethics Committee for Scientific Research at Faculty of Psychology, University of Warsaw (Komisja Etyki Badań Naukowych Wydziału Psychologii Uniwersytetu Warszawskiego), Warsaw, Poland. All study participants provided written informed consent.

\section{Consent for publication}

Not applicable

\section{Availability of data and materials}

Self-generated questionnaires are available on https://osf.io/qj9mf/.

\section{Competing interests}

The authors declare that they have no competing interests. RK receives advisory honoraria from JoyVentures, Herzlia, Israel.

\section{Funding}

This project has received funding from the European Union's Horizon 2020 research and innovation program under Grant Agreement numbers 777084 (DynaMORE project) and 101016127 (RESPOND project), Deutsche Forschungsgemeinschaft (DFG Grant CRC 1193, subprojects B01, C01, C04, Z03), the German Federal Ministry for Education and Research (BMBF) as part of the Network for University Medicine under Grant number 01KX2021 (CEOsys and EViPan projects), and the State of Rhineland-Palatinate, Germany (MARP program, DRZ program, Leibniz Institute for Resilience Research). A.R. is supported by Studienstiftung des deutschen Volkes. The funding agencies had no part in study design, collection, management, analysis, and interpretation of data, writing of the report, and the decision to submit the report for publication. 


\section{Authors' contributions}

Drafting the manuscript: CW, IV, JMCL, RK, HW

Conception \& Design of the study: RK, HW, IV, AA-V, BK, DK, EH, HB, IM-G, JT, KR, KY, MM, OT, SP, TH, WdR

Acquisition of data: CW, JMCL, SB, NM, LP, ZR, AR, AU, MZ, GK, JW, KY

Critical editing and revision of the manuscript: All authors

All authors have approved of the submitted version of this manuscript.

All authors have agreed to be personally accountable for the author's own contributions and to ensure that questions related to the accuracy or integrity of any part of the work, even ones in which the author was not personally involved, are appropriately investigated, resolved, and the resolution documented in the literature.

\section{Acknowledgements}

We thank S. Stöber and N. Donner for their help with project administration. For their help with study conduct, we thank the following people: At Charité: A. Dimitrov, V. Wessel, P. Eisenhauer, L. Schmidt, E. Hodapp, C. Sachs, N. Hoerner and D. Alkhoury; in Mainz: S. Paleske, K. Aschenbroich, L. Dressler, C. Sakas Gandullo, A. Peschel, C. Walter, M. Weber, J. Piloth: in Nijmegen: J. Asbreuk, E. Hermsen, Y. Schwarze; in Tel Aviv: A. Lerner, I. David, G. Shahaf, S.Toledano and L. Andrawus; in Warsaw: J. Mituniewicz, N. Robak, M. Górecki, J. Szurnicka, P. Berg. 


\section{References}

1. Olesen J, Gustavsson A, Svensson M, Wittchen H-U, Jönsson B. The economic cost of brain disorders in Europe. European Journal of Neurology. 2012;19:155-62.

2. Vos T, Flaxman AD, Naghavi M, Lozano R, Michaud C, Ezzati M, et al. Years lived with disability (YLDs) for 1160 sequelae of 289 diseases and injuries 1990-2010: a systematic analysis for the Global Burden of Disease Study 2010. The Lancet. 2012;380:2163-96.

3. GBD 2016 DALYs and HALE Collaborators. Global, regional, and national disability-adjusted life-years (DALYs) for 333 diseases and injuries and healthy life expectancy (HALE) for 195 countries and territories, 1990-2016: a systematic analysis for the Global Burden of Disease Study 2016. Lancet. 2017;390:1260-344.

4. Gradus JL. Prevalence and prognosis of stress disorders: a review of the epidemiologic literature. Clin Epidemiol. 2017;9:251-60.

5. Walker ER, McGee RE, Druss BG. Mortality in Mental Disorders and Global Disease Burden Implications. JAMA Psychiatry. 2015;72:334-41.

6. Gustavson K, Knudsen AK, Nesvåg R, Knudsen GP, Vollset SE, Reichborn-Kjennerud T. Prevalence and stability of mental disorders among young adults: findings from a longitudinal study. BMC Psychiatry. 2018;18:65.

7. Antonovsky A. Unraveling the Mystery of Health: How People Manage Stress and Stay Well. 1st edition. San Francisco: Jossey-Bass Inc.,U.S.; 1987.

8. Bonanno GA, Westphal M, Mancini AD. Resilience to Loss and Potential Trauma. Annu Rev Clin Psychol. 2011;7:511-35.

9. Kalisch R, Müller MB, Tüscher O. A conceptual framework for the neurobiological study of resilience. Behavioral and Brain Sciences. 2015;38.

10. Bonanno GA, Romero SA, Klein SI. The temporal elements of psychological resilience: An integrative framework for the study of individuals, families, and communities. Psychological Inquiry. 2015;26:139-69.

11. Kalisch R, Baker DG, Basten U, Boks MP, Bonanno GA, Brummelman E, et al. The resilience framework as a strategy to combat stress-related disorders. Nature Human Behaviour. 2017;1:784-90.

12. Kalisch R, Cramer AOJ, Binder H, Fritz J, Leertouwer lj, Lunansky G, et al. Deconstructing and Reconstructing Resilience: A Dynamic Network Approach. Perspect Psychol Sci. 2019;14:765-77.

13. Kalisch R, Köber G, Binder H, Ahrens KF, Basten U, Chmitorz A, et al. The Frequent Stressor and Mental Health Monitoring-Paradigm: A Proposal for the Operationalization and Measurement of Resilience and the Identification of Resilience Processes in Longitudinal Observational Studies. Frontiers in Psychology. 2021;12:3377.

14. Ungar M. Resilience and Culture: The Diversity of Protective Processes and Positive Adaptation. In: Theron LC, Liebenberg L, Ungar M, editors. Youth Resilience and Culture: Commonalities and Complexities. Dordrecht: Springer Netherlands; 2015. p. 37-48.

15. Boals A, Schuettler D, Southard-Dobbs S. Construing trauma as a double-edged sword: how narrative components of autobiographical memory relate to devastation and growth from trauma. In: Berntsen D, Watson LA, editors. Clinical Perspectives on Autobiographical Memory. Cambridge: Cambridge University Press; 2015. p. 65-84.

16. Luthar SS, Cicchetti D, Becker B. The construct of resilience: a critical evaluation and guidelines for future work. Child Dev. 2000;71:543-62.

17. Ayash S, Schmitt U, Lyons DM, Müller MB. Stress inoculation in mice induces global resilience. TransI Psychiatry. 2020;10:1-8. 
18. Mancini AD. When acute adversity improves psychological health: A social-contextual framework. Psychol Rev. 2019;126:486-505.

19. Seery MD, Leo RJ, Lupien SP, Kondrak CL, Almonte JL. An Upside to Adversity?: Moderate Cumulative Lifetime Adversity Is Associated With Resilient Responses in the Face of Controlled Stressors. Psychol Sci. 2013;24:1181-9.

20. Boks MP, van Mierlo HC, Rutten BPF, Radstake TRDJ, De Witte L, Geuze E, et al. Longitudinal changes of telomere length and epigenetic age related to traumatic stress and post-traumatic stress disorder. Psychoneuroendocrinology. 2015;51:506-12.

21. Breen MS, Maihofer AX, Glatt SJ, Tylee DS, Chandler SD, Tsuang MT, et al. Gene networks specific for innate immunity define post-traumatic stress disorder. Molecular Psychiatry. 2015;20:1538-45.

22. Krishnan V, Han M-H, Graham DL, Berton O, Renthal W, Russo SJ, et al. Molecular adaptations underlying susceptibility and resistance to social defeat in brain reward regions. Cell. 2007;131:391-404.

23. Friedman AK, Walsh JJ, Juarez B, Ku SM, Chaudhury D, Wang J, et al. Enhancing depression mechanisms in midbrain dopamine neurons achieves homeostatic resilience. Science. 2014;344:313-9.

24. Wang M, Perova Z, Arenkiel BR, Li B. Synaptic Modifications in the Medial Prefrontal Cortex in Susceptibility and Resilience to Stress. J Neurosci. 2014;34:7485-92.

25. Maier SF. Behavioral control blunts reactions to contemporaneous and future adverse events: Medial prefrontal cortex plasticity and a corticostriatal network. Neurobiology of Stress. 2015;1:12-22.

26. Masten AS. Multisystem Resilience: Pathways to an Integrated Framework. Research in Human Development. 2021;18:153-63.

27. Reavley N, Jorm AF. Prevention and early intervention to improve mental health in higher education students: a review. Early Intervention in Psychiatry. 2010;4:132-42.

28. Eisenberg D, Hunt J, Speer N. Help seeking for mental health on college campuses: review of evidence and next steps for research and practice. Harv Rev Psychiatry. 2012;20:222-32.

29. Frajerman A, Morvan $Y$, Krebs M-O, Gorwood P, Chaumette B. Burnout in medical students before residency: A systematic review and meta-analysis. Eur Psychiatry. 2019;55:36-42.

30. Gould J. Mental health: Stressed students reach out for help. Nature. 2014;512:223-4.

31. Herbst U, Voeth M, Eidhoff AT, Müller M, Stief S. Studierendenstress in Deutschland - eine empirische Untersuchung. 2016.

32. Rotenstein LS, Ramos MA, Torre M, Segal JB, Peluso MJ, Guille C, et al. Prevalence of Depression, Depressive Symptoms, and Suicidal Ideation Among Medical Students. JAMA. 2016;316:2214-36.

33. Tupler LA, Hong JY, Gibori R, Blitchington TF, Krishnan KRR. Suicidal Ideation and Sex Differences in Relation to 18 Major Psychiatric Disorders in College and University Students: Anonymous Web-Based Assessment. The Journal of Nervous and Mental Disease. 2015;203:269-78.

34. Cochrane R, Robertson A. The life events inventory: A measure of the relative severity of psycho-social stressors. Journal of Psychosomatic Research. 1973;17:135-9.

35. Goldberg DP, Gater R, Sartorius N, Ustun TB, Piccinelli M, Gureje O, et al. The validity of two versions of the GHQ in the WHO study of mental illness in general health care. Psychol Med. 1997;27:191-7. 
36. Veer IM, Riepenhausen A, Zerban M, Wackerhagen C, Puhlmann LMC, Engen $\mathrm{H}$, et al. Psycho-social factors associated with mental resilience in the Corona lockdown. Translational Psychiatry. 2021;11:1-11.

37. Kampa M, Schick A, Yuen K, Sebastian A, Chmitorz A, Saase V, et al. A Combined Behavioral and Neuroimaging Battery to Test Positive Appraisal Style Theory of Resilience in Longitudinal Studies. bioRxiv. 2018;:470435.

38. Kampa M, Schick A, Sebastian A, Wessa M, Tüscher O, Kalisch R, et al. Replication of fMRI group activations in the neuroimaging battery for the Mainz Resilience Project (MARP). Neuroimage. 2020;204:116223.

39. Chmitorz A, Neumann RJ, Kollmann B, Ahrens KF, Öhlschläger S, Goldbach N, et al. Longitudinal determination of resilience in humans to identify mechanisms of resilience to modern-life stressors: the longitudinal resilience assessment (LORA) study. Eur Arch Psychiatry Clin Neurosci. 2021;271:1035-51.

40. Bögemann S, Kalisch R, Hermans E, Puhlmann L. DynaCORE-L: The DynaMORE longitudinal study on psychological resilience to the mental health consequences of the Corona crisis. 2020. https://osf.io/d6wgr/. Accessed 12 Jan 2021.

41. Overbeek L. Rationale and design of the Healthy Brain Study: an accessible resource for understanding the human brain and how it dynamically and individually operates in its biosocial context. 2021.

42. Köber G, Pooseh S, Engen H, Chmitorz A, Kampa M, Schick A, et al. Individualizing deep dynamic models for psychological resilience data. medRxiv. 2020;:2020.08.18.20177113.

43. Schüler K, Fritz J, Dorfschmidt L, Harmelen A-LV, Strömer E, Wessa M. Psychological Network Analysis of General Self-Efficacy in High vs Low Resilient Functioning Healthy Adults. 2020.

44. Ungar M. Social ecological complexity and resilience processes. Behavioral and Brain Sciences. 2015;38.

45. Streit F, Haddad L, Paul T, Frank J, Schäfer A, Nikitopoulos J, et al. A functional variant in the neuropeptide $S$ receptor 1 gene moderates the influence of urban upbringing on stress processing in the amygdala. Stress. 2014;17:352-61.

46. Sandner $M$, Lois $G$, Streit $F$, Zeier $P$, Kirsch $P$, Wüst $S$, et al. Investigating individual stress reactivity: High hair cortisol predicts lower acute stress responses. Psychoneuroendocrinology. 2020;118:104660.

47. Hariri AR, Mattay VS, Tessitore A, Kolachana B, Fera F, Goldman D, et al. Serotonin transporter genetic variation and the response of the human amygdala. Science. 2002;297:400-3.

48. Wackerhagen C, Wüstenberg T, Mohnke S, Erk S, Veer IM, Kruschwitz JD, et al. Influence of Familial Risk for Depression on Cortico-Limbic Connectivity during Implicit Emotional Processing. Neuropsychopharmacology. 2017;42:1729-38.

49. Wackerhagen C, Veer IM, Erk S, Mohnke S, Lett TA, Wüstenberg T, et al. Amygdala functional connectivity in major depression - disentangling markers of pathology, risk and resilience. Psychological Medicine. 2019;:1-11.

50. Bornstein RA. Normative data on selected neuropsychological measures from a nonclinical sample. Journal of Clinical Psychology. 1985;41:651-9.

51. Tombaugh TN. Trail Making Test A and B: Normative data stratified by age and education. Archives of Clinical Neuropsychology. 2004;19:203-14.

52. Ryan JJ, Lopez SJ. Wechsler Adult Intelligence Scale-III. In: Dorfman WI, Hersen M, editors. Understanding Psychological Assessment. Boston, MA: Springer US; 2001. p. 19-42. 
53. Armbruster DJN, Ueltzhöffer K, Basten U, Fiebach CJ. Prefrontal Cortical Mechanisms Underlying Individual Differences in Cognitive Flexibility and Stability. Journal of Cognitive Neuroscience. 2012;24:2385-99.

54. Kraft D, Rademacher L, Fiebach C. Cognitive, affective and feedback-based flexibility disentangling shared and different aspects of psychological flexibility. 2018. https://doi.org/10.17605/OSF.IO/8F4CN.

55. Feinberg DA, Moeller S, Smith SM, Auerbach E, Ramanna S, Glasser MF, et al. Multiplexed Echo Planar Imaging for Sub-Second Whole Brain FMRI and Fast Diffusion Imaging. PLOS ONE. 2010;5:e15710.

56. Littlejohns TJ, Holliday J, Gibson LM, Garratt S, Oesingmann N, Alfaro-Almagro F, et al. The UK Biobank imaging enhancement of 100,000 participants: rationale, data collection, management and future directions. Nat Commun. 2020;11:2624.

57. Knutson B, Adams CM, Fong GW, Hommer D. Anticipation of increasing monetary reward selectively recruits nucleus accumbens. J Neurosci. 2001;21:RC159-RC159.

58. de Voogd LD, Murray YPJ, Barte RM, van der Heide A, Fernández G, Doeller CF, et al. The role of hippocampal spatial representations in contextualization and generalization of fear. Neurolmage. 2020;206:116308.

59. Kanske P, Heissler J, Schönfelder S, Bongers A, Wessa M. How to Regulate Emotion? Neural Networks for Reappraisal and Distraction. Cerebral Cortex. 2011;21:1379-88.

60. Wessa M, Kanske P, Neumeister P, Bode K, Heissler J, Schönfelder S. EmoPics: Subjektive und psychophysiologische Evaluation neuen Bildmaterials für die klinisch-bio-

psychologische Forschung. Zeitschrift für Klinische Psychologie und Psychotherapie. 2010;39 Suppl. 1/11:77.

61. Lang PJ, Bradley MM, Cuthbert BN. International affective picture system (IAPS): Affective ratings of pictures and instruction manual. Technichal Report A-8. University of Florida, Gainesville, FL; 2008.

62. Ekman P, Friesen WV. Pictures of Facial Affect. Palo Alto: Consulting Psychologists Press; 1976.

63. Akdeniz C, Tost H, Streit F, Haddad L, Wüst S, Schäfer A, et al. Neuroimaging evidence for a role of neural social stress processing in ethnic minority-associated environmental risk. JAMA Psychiatry. 2014;71:672-80.

64. Lederbogen F, Kirsch P, Haddad L, Streit F, Tost H, Schuch P, et al. City living and urban upbringing affect neural social stress processing in humans. Nature. 2011;474:498-501.

65. Dahm A-S, Schmierer P, Veer IM, Streit F, Görgen A, Kruschwitz J, et al. The burden of conscientiousness? Examining brain activation and cortisol response during social evaluative stress. Psychoneuroendocrinology. 2017;78:48-56.

66. Sheehan DV, Lecrubier Y, Sheehan KH, Amorim P, Janavs J, Weiller E, et al. The MiniInternational Neuropsychiatric Interview (M.I.N.I.): the development and validation of a structured diagnostic psychiatric interview for DSM-IV and ICD-10. J Clin Psychiatry. 1998;59 Suppl 20:22-33;quiz 34-57.

67. Lewis SJ, Heaton KW. Stool form scale as a useful guide to intestinal transit time. Scand J Gastroenterol. 1997;32:920-4.

68. Chmitorz A, Kurth K, Mey LK, Wenzel M, Lieb K, Tüscher O, et al. Assessment of Microstressors in Adults: Questionnaire Development and Ecological Validation of the Mainz Inventory of Microstressors. JMIR Mental Health. 2020;7:e14566.

69. Cohen S, Kamarck T, Mermelstein R. A global measure of perceived stress. Journal of Health and Social Behavior. 1983;24:385-96. 
70. Roberti JW, Harrington LN, Storch EA. Further Psychometric Support for the 10-Item Version of the Perceived Stress Scale. Journal of College Counseling. 2006;9:135-47.

71. Derogatis L. SCL-90: Administration, scoring and procedure manual-I for the R (revised) version. Baltimore: Johns Hopkins University School of Medicine; 1977.

72. Üstün TB, Chatterji S, Kostanjsek N, Rehm J, Kennedy C, Epping-Jordan J, et al. Developing the World Health Organization Disability Assessment Schedule 2.0. Bull World Health Organ. 2010;88:815-23.

73. Smith BW, Dalen J, Wiggins K, Tooley E, Christopher P, Bernard J. The brief resilience scale: Assessing the ability to bounce back. International Journal of Behavioral Medicine. 2008;15:194-200.

74. Garnefski N, Kraaij V. Cognitive emotion regulation questionnaire - development of a short 18-item version (CERQ-short). Personality and Individual Differences. 2006;41:1045-53.

75. Carver CS. You want to measure coping but your protocol's too long: consider the brief COPE. Int J Behav Med. 1997;4:92-100.

76. Luszczynska A, Gutiérrez-Doña B, Schwarzer R. General self-efficacy in various domains of human functioning: Evidence from five countries. International Journal of Psychology. 2005;40:80-9.

77. Kovaleva A. The IE-4: construction and validation of a short scale for the assessment of locus of control. Köln [Mannheim]: GESIS - Leibniz Inst. for the Social Sciences; 2012.

78. Chiesi F, Galli S, Primi C, Borgi PI, Bonacchi A. The Accuracy of the Life Orientation TestRevised (LOT-R) in Measuring Dispositional Optimism: Evidence From Item Response Theory Analyses. Journal of Personality Assessment. 2013;95:523-9.

79. Korner A, Czajkowska Z, Albani C, Drapeau M, Geyer M, Braehler E. Efficient and valid assessment of personality traits: population norms of a brief version of the NEO Five-Factor Inventory (NEO-FFI). Arch Psych Psych. 2015;17:21-32.

80. Kocalevent R-D, Berg L, Beutel ME, Hinz A, Zenger M, Härter M, et al. Social support in the general population: standardization of the Oslo social support scale (OSSS-3). BMC Psychology. 2018;6:31.

81. Maor M, Gurion B, Ben-Itzhak S, Bluvstein I. The Psychological Flexibility Questionnaire (PFQ): Development, Reliability and Validity. 2014.

82. Olthuis JV, Watt MC, Stewart SH. Anxiety Sensitivity Index (ASI-3) subscales predict unique variance in anxiety and depressive symptoms. Journal of Anxiety Disorders. 2014;28:11524.

83. Walkup H, Abbott RD. Cross-Validation of Item Selection on the Bem Sex Role Inventory. Applied Psychological Measurement. 1978;2:63-71.

84. Bem SL. Bem Sex Role Inventory - PsycNET. 1974.

85. Shields SA, Mallory ME, Simon A. The Body Awareness Questionnaire: Reliability and Validity. Journal of Personality Assessment. 1989;53:802-15.

86. Connor KM, Davidson JRT. Development of a new resilience scale: The Connor-Davidson Resilience Scale (CD-RISC). Depression and Anxiety. 2003;18:76-82.

87. Rizvi SJ, Quilty LC, Sproule BA, Cyriac A, Michael Bagby R, Kennedy SH. Development and validation of the Dimensional Anhedonia Rating Scale (DARS) in a community sample and individuals with major depression. Psychiatry Res. 2015;229:109-19.

88. Gross JJ. Emotion regulation: Affective, cognitive, and social consequences.

Psychophysiology. 2002;39:281-91. 
89. Teicher MH, Parigger A. The 'Maltreatment and Abuse Chronology of Exposure' (MACE) Scale for the Retrospective Assessment of Abuse and Neglect During Development. PLoS One. 2015;10.

90. Adler NE, Epel ES, Castellazzo G, Ickovics JR. Relationship of subjective and objective social status with psychological and physiological functioning: preliminary data in healthy white women. Health Psychol. 2000;19:586-92.

91. Brinker JK, Dozois DJA. Ruminative thought style and depressed mood. Journal of Clinical Psychology. 2009;65:1-19.

92. Torrubia R, Ávila C, Moltó J, Caseras X. The Sensitivity to Punishment and Sensitivity to Reward Questionnaire (SPSRQ) as a measure of Gray's anxiety and impulsivity dimensions. Personality and Individual Differences. 2001;31:837-62.

93. Spielberger CD, Gorsuch RL, Lushene RE. Manual for the State-Trait Anxiety Inventory. Palo Alto, CA: Consulting Psychologists Press; 1970.

94. Parker JDA, Taylor GJ, Bagby RM. The 20-Item Toronto Alexithymia Scale: III. Reliability and factorial validity in a community population. Journal of Psychosomatic Research. 2003;55:269-75. 


\section{Tables}

\begin{tabular}{|c|c|c|}
\hline Nr. & Criterion & Format \\
\hline 1 & Age between 18 and 25 (18 and 27 at Tel Aviv University). & Online \\
\hline 2 & 3 or more life events rated as burdening. & Online \\
\hline 3 & GHQ-28 score of 20 or higher. & Online \\
\hline 4 & Body mass index between 18 and 27. & Online \\
\hline 5 & No hormonal treatment and no consumption of / treatment with steroids. & Online \\
\hline 6 & $\begin{array}{l}\text { Proficiency in the official language of the country of study enrollment (minimum } \\
\text { level of } C 1 \text { in the Common European Framework of Reference for Languages). }\end{array}$ & Online \\
\hline 7 & $\begin{array}{l}\text { Eligibility to participate in ecological physiological assessment using a wearable } \\
\text { device (no skin disease in the wrist or chest area and no medical condition that } \\
\text { increases risk of infection through electrodes, no medication with phototoxic side } \\
\text { effects). }\end{array}$ & Online \\
\hline 8 & $\begin{array}{l}\text { Eligibility to participate in the fear conditioning task (no skin allergy or allergy to } \\
\text { adhesive electrodes). }\end{array}$ & Online \\
\hline 9 & $\begin{array}{l}\text { No lifetime diagnosis of any severe mental or organic disorder that affects } \\
\text { neurodevelopment due to its pathological mechanism or treatment (e.g., } \\
\text { schizophrenia, bipolar disorder, anorexia/bulimia nervosa, attention deficit } \\
\text { hyperactivity disorder, autism spectrum disorder, meningitis, epilepsy, multiple } \\
\text { sclerosis, stroke, brain cancer, brain concussion, or coma). }\end{array}$ & $\begin{array}{l}\text { Online + } \\
\text { Interview }\end{array}$ \\
\hline 10 & $\begin{array}{l}\text { Eligibility for undergoing the functional magnetic resonance imaging protocol } \\
\text { (normal or corrected-to-normal eyesight, no hearing impairment, no claustrophobia, } \\
\text { no non-removable ferromagnetic metal in or at the body, not pregnant, no large } \\
\text { tattoo in head or neck area). }\end{array}$ & $\begin{array}{l}\text { Online + } \\
\text { interview }\end{array}$ \\
\hline 11 & $\begin{array}{l}\text { No diagnosis within } 9 \text { months before inclusion of any mental disorder other than a } \\
\text { mild depressive episode (ICD F32.1), tobacco abuse/dependence (ICD F12), or } \\
\text { substance abuse, as assessed by trained psychologists using the Mini-International } \\
\text { Neuropsychiatric Interview (M.I.N.I. [66]). }\end{array}$ & Interview \\
\hline 12 & $\begin{array}{l}\text { No consumption of any psychoactive drug or substance up to } 4 \text { weeks prior to the } \\
\text { first psychological assessment and to the MRI assessment. }\end{array}$ & Interview \\
\hline 13 & $\begin{array}{l}\text { The participant has received all relevant information about the study, is able to } \\
\text { obtain full insight and is fully contractually capable, is willing and able to comply } \\
\text { with the protocol and agrees to participate by giving written consent. }\end{array}$ & Interview \\
\hline
\end{tabular}

Table 1. List of inclusion criteria and format in which they were assessed. Participants who were found eligible in criteria 1-10 in the anonymous online screening were invited to an on-site interview to confirm/check eligibility for the criteria $9-13$, to receive written and verbal information about the study and to provide written informed consent. 
Procedure Steps at Baseline Days 1 and 2

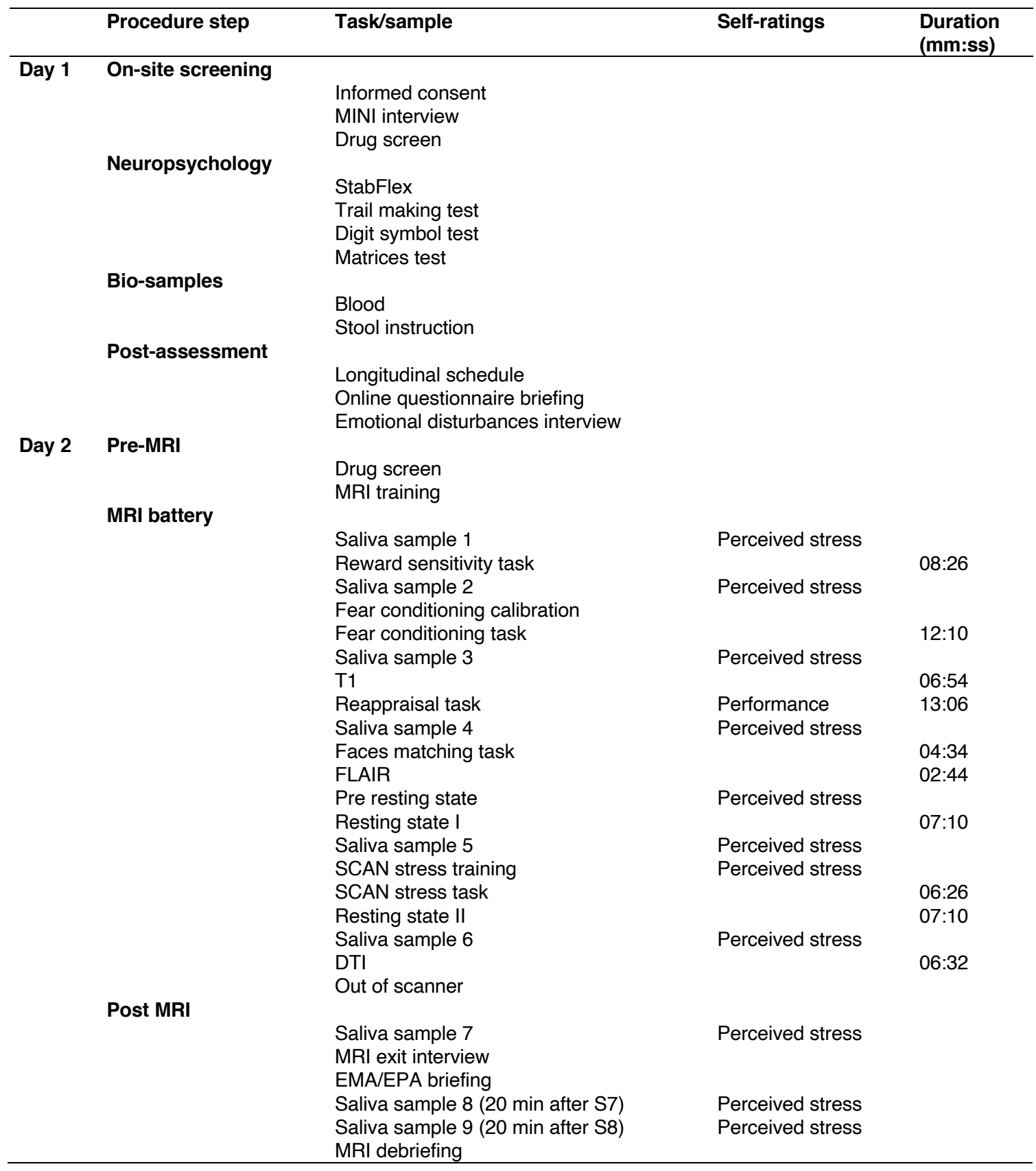

Table 2. Procedure steps at baseline days 1 and 2. Note that, before each fMRI sequence, a field map scan was acquired. The total duration of the imaging battery was about 2 hours. Abbreviations: DTI, Diffusion Tensor Imaging; EMA, Ecological Momentary Assessment; EPA, Ecological Physiology Assessment; FLAIR - Fluid-Attenuated Inversion Recovery; MRI, Magnetic Resonance Imaging; S, saliva sample; T1, T1- weighted image. 


\begin{tabular}{|c|c|c|c|c|c|c|c|c|c|c|c|c|c|c|c|c|c|c|c|c|c|c|c|c|c|c|c|c|c|c|c|c|}
\hline & & & & Bas & line & & & & & & & & & & ase I & & & & & & & & & & & & & Phas & se II & & & \\
\hline & & & Scree- & & & & M1 & & & M2 & & & M3 & & & M4 & & & M5 & & & M6 & & & M7 & 17 & & $\mathbf{M}$ & & & M9 & \\
\hline & & & ning & d1 & d2 & w1 & & w3 w4 & w1 & & w3 w4 & w1 & w2 & N3 & $4 \mathrm{w} 1$ & w2 v & w3 w4 & w1 & $\mathrm{w2}$ & w3 w4 & w1 y & w2 ws & N3 w4 & 4 w1 & w2 & w3 $w$ & & v1 w2 & w3 & & w1 w2 w3 w & \\
\hline 흐 & Inclusion crit & teria & $\mathbf{x}$ & & & & & & & & & & & & & & & & & & & & & & & & & & & & & \\
\hline$\stackrel{0}{=}$ & MINI Intervie & & & $\mathbf{x}$ & & & & & & & & & & & & & & & & & & & & & & & & & & & & \\
\hline$\stackrel{\mathscr{S}}{\underline{E}}$ & Drug screeni & ing & & $\mathbf{x}$ & $\mathbf{x}$ & & & & & & & & & & & & & & & & & & & & & & & & & & & \\
\hline & Online Questi & ionnaires & & & & & & & & & & & & & & & & & & & & & & & & & & & & & & \\
\hline & $\mathrm{GHQ}-28$ & $\begin{array}{l}\text { General Health } \\
\text { Questionnaire }\end{array}$ & $\mathbf{x}$ & & & $\mathbf{x}$ & & $\mathbf{x}$ & $\mathbf{x}$ & & $\mathbf{x}$ & $\mathbf{x}$ & & $\mathrm{x}$ & $x$ & & $x$ & $\mathbf{x}$ & & $x$ & $\mathbf{x}$ & & $\mathrm{x}$ & & & $x$ & & & $\mathbf{x}$ & & $\mathbf{x}$ & \\
\hline 艾 & MIMIS & \begin{tabular}{|l|} 
Mainz Inventory of \\
Microstressors
\end{tabular} & & & & $\mathbf{x}$ & & $\mathbf{x}$ & $\mathbf{x}$ & & $\mathbf{x}$ & $\mathbf{x}$ & & $\mathbf{x}$ & $\mathbf{x}$ & & $\mathbf{x}$ & $\mathbf{x}$ & & $\mathbf{x}$ & $\mathbf{x}$ & & $x$ & & & $\mathbf{x}$ & & & $\mathbf{x}$ & & $\mathbf{x}$ & \\
\hline $\begin{array}{l}\mathbb{\pi} \\
\mathbb{\pi} \\
\tilde{\pi}\end{array}$ & coV Stress & Covid-related Stressors & & & & $\mathbf{x}$ & & $\mathbf{x}$ & & & $\mathbf{x}$ & & & $\mathbf{x}$ & & & $\mathbf{x}$ & & & $\mathbf{x}$ & & $x$ & $\mathbf{x}$ & & & $\mathbf{x}$ & & & $\mathbf{x}$ & & $\mathbf{x}$ & \\
\hline $\bar{c}$ & LEQ & Life Event Questionnaire & $\mathbf{x}$ & & & $\mathbf{x}$ & & & & & & & & $\mathbf{x}$ & & & & & & & & $\mathbf{x}$ & $\mathbf{x}$ & & & & & & & & $\mathbf{x}$ & \\
\hline d & SCL-90-R & $\begin{array}{l}\text { Symptom Checklist } 90 \\
\text { Revised }\end{array}$ & & & & $\mathbf{x}$ & & & & & & & & $\mathbf{x}$ & & & & & & & & $\mathbf{x}$ & $x$ & & & & & & & & $\mathbf{x}$ & \\
\hline & WHO-DAS & \begin{tabular}{|l} 
WHO Disability \\
Assessment Scale
\end{tabular} & & & & $\mathbf{x}$ & & & & & & & & $\mathrm{x}$ & & & & & & & & $\mathbf{x}$ & $x$ & & & & & & & & $\mathbf{x}$ & \\
\hline 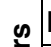 & Neuropsychol & logical battery & & $\mathbf{x}$ & & & & & & & & & & & & & & & & & & & & & & & & & & & & \\
\hline : & Neuroimaging & g battery & & & $\mathbf{x}$ & & & & & & & & & & & & & & & & & & & & & & & & & & & \\
\hline 吾 & Online Questi & ionnaires & & & & & & & & & & & & & & & & & & & & & & & & & & & & & & \\
\hline 递 & PASSp & $\begin{array}{l}\text { Positive Appraisal Style } \\
\text { Scale - process-based }\end{array}$ & & & & $\mathbf{x}$ & & $x$ & $\mathbf{x}$ & & $x$ & $\mathbf{x}$ & & $x$ & $\mathbf{x}$ & & $\mathbf{x}$ & $\mathbf{x}$ & & $x$ & $\mathbf{x}$ & $\mathbf{x}$ & $\mathbf{x}$ & & & $\mathbf{x}$ & & & $\mathbf{x}$ & & $\mathbf{x}$ & \\
\hline टे & Other primar & ry resilience and risk factors & & & & $\mathbf{x}$ & & & & & & & & & & & & & & & & & & & & & & & & & $\mathbf{x}$ & \\
\hline$\stackrel{\Xi}{\Xi}$ & Secondary re & esilience and risk factors & & & & $\mathbf{x}$ & & & & & & & & & & & & & & & & & & & & & & & & & & \\
\hline 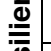 & Sports and $\mathrm{m}$ & nental activities & & & & & & & & & & & & $\mathbf{x}$ & & & & & & & & $\mathbf{x}$ & $\mathbf{x}$ & & & & & & & & $\mathbf{x}$ & \\
\hline $\mathbb{8}$ & Bio-samples & & & & & & & & & & & & & & & & & & & & & & & & & & & & & & & \\
\hline$\overline{\underline{\sigma}}$ & EDTA Blood & DNA/DNA-methylation & & $\mathbf{x}$ & & & & & & & & & & & & & & & & & & $\mathbf{x}$ & $x$ & & & & & & & & & \\
\hline 节 & Stool & Microbiome & & & & $\mathbf{x}$ & & & & & & & & $\mathbf{x}$ & & & & & & & & $x$ & $x$ & & & & & & & & & \\
\hline 。ै & Saliva & $\begin{array}{l}\text { Cortisol during MRI } \\
\text { assessment }\end{array}$ & & & $\mathbf{x}$ & & & & & & & & & & & & & & & & & & & & & & & & & & & \\
\hline 0 & Ambulatory A & Assessments & & & & & & & & & & & & & & & & & & & & & & & & & & & & & & \\
\hline 总 & EMA & $\begin{array}{l}\text { Ecological Momentary } \\
\text { Assessment }\end{array}$ & & & & & $\mathbf{x}$ & & & $\mathbf{x}$ & & & $\mathbf{x}$ & & & $x$ & & & $\mathbf{x}$ & & & $\mathbf{x}$ & & & & & & & & & & \\
\hline ฉ̀ & EPA & $\begin{array}{l}\text { Ecological Physiological } \\
\text { Assessment }\end{array}$ & & & & & $\mathbf{x}$ & & & $\mathbf{x}$ & & & $\mathbf{x}$ & & & $x$ & & & $\mathbf{x}$ & & & $\mathbf{x}$ & & & & & & & & & & \\
\hline
\end{tabular}

Table 3. Overview of the measures used and the days (d), weeks (w) and months (M) from baseline, at which they are assessed (x). Resilience and risk factors (RFs) are grouped into primary and secondary RFs. Primary RFs are of main interest in the current study based on previous findings and theoretical background of our consortium [9, 36, 40], while secondary RFs are based on hypotheses drawn from the literature. 
28 stressful life events (e.g., death of a friend or family member, separation or divorce of the parents, illness or injury). For each event, participants indicate whether and at what age it has occurred and how positive or burdensome it has been experienced [34].

List of COVID-related stressors (COV stress)

A list of 23 stressors specific to the COVID-pandemic (e.g., being at increased risk for an infection, loss of socia contact, having COVID symptoms, etc.), for which participants report whether the situation occurred and how burdensome it was perceived on a 5-point scale. The list was self-developed in March 2020 for the DynaCORE studies on psychological resilience during the COVID-pandemic [36, 40].

Mainz Inventory of

Microstressors (MMIS)

58 minor stressors of daily life (e.g. loss or displacement of an object, conflict, bad weather, traffic). Participants report whether the events have occurred and how straining they were experienced on a 5-point scale [68].

\section{Mental health status}

General Health Questionnaire (GHQ-28)

Perceived Stress Scale (PSS-10)

Revised Symptom Checklist 90 (SCL-90-R)

WHO Disability Assessment Schedule (WHODAS 2.0)

\section{Questionnaires used to assess prim}

Brief Resilience Scale (BRS)

Cognitive Emotion Regulation Questionnaire (CERQ short)

Coping Orientation to Problems Experienced questionnaire (Brief COPE)

General Positive Appraisal Style Scale (gPASS)

General Self Efficacy Scale (GSE)

Internal External Locus of

Control-4 (IE-4)

Life Orientation Test - Revised (LOT-R)

NEO-Neuroticism
Symptoms of anxiety, depression, insomnia, social problems as well as somatic symptoms. This inventory is designed to capture the inability to carry out normal functions and the appearance of new and distressing phenomena in the general population, 28 items [35]

\section{overloaded, 10 items [69, 70].}

Psychological distress in terms of nine primary symptom dimensions including somatization, obsessive-compulsive, interpersonal sensitivity, depression, anxiety, hostility, phobic anxiety, paranoid ideation, and psychoticism, 90 items [71].

Functioning and disability in accordance with the International Classification of Functioning, Disability and Health, 12 items [72].

\section{resilience and risk factors}

The subjective ability to cope with and recover from stress, 10 items [73].

Different strategies of emotion regulation such as self-blame, other-blame, rumination, catastrophizing, positive refocusing, planning, positive reappraisal, putting into perspective, and acceptance, 18 items [74].

Emotion regulation strategies such as self-distraction, active coping, denial, substance use, use of emotional support, use of instrumental support, behavioral disengagement, venting, positive reframing, planning, humor, acceptance, religion, and self-blame, 28 items [75].

General style of positive appraisal in stressful situations, focusing on appraisal contents, 29 items (self-developed). Validation and condensation into a Positive Appraisal Style Scale - content-based (PASSc) in progress.

Perceived ability to cope with a variety of difficult demands in life, 10 items [76].

Degree to which individuals perceive themselves the outcomes of their behavior to be determined by their own actions or by forces outside of their control 4 items, 4 items [77].

Dispositional optimism and pessimism, 10 items [78].

Neuroticism scale of the NEO Five Factor Inventory (NEO-FFI), 12 items [79]. 
Oslo 3 Item Social Support Scale (OSS-3)

Positive Appraisal Style Scale process-based (PASSp)
Psychological Flexibility Questionnaire (PFQ)

\section{Degree to which} supportive [80]

Assessment of positive appraisal style focusing on cognitive processes that generate positive appraisal contents in stressful situations (as opposed to assessment of the resulting appraisal contents themselves, as in PASSc above). Referred to as PASS in Veer et al. (2021). PASSp includes items of the Brief COPE, the CERQ short, as well as two own-formulated items on distancing (detachment). In a combined principal component analysis of COPE, CERQ, and distancing in our previous studies MARP and LORA, we consistently identified three components, of which one was readily interpretable as indexing processes leading to positive appraisals. After removing overlapping $\mathrm{CERQ/COPE}$ subscales, PASSp includes the scales distancing (dis; own-formulated), positive reappraisal (pra; $\mathrm{CERQ}$ ), acceptance (acc; CERQ), putting into perspective (per; CERQ), refocus on planning (rfp; CERQ), positive refocusing (prf; CERQ), and humor (hum; COPE).

Subjective psychological flexibility, assessed via five factors including positive perception of change, characterization of the self as flexible, self-characterization as open and innovative, a perception of reality as dynamic and changing, and a perception of reality as multifaceted, 20 items [81].

\section{Questionnaires used to assess secondary resilience and risk factors}

Anxiety Sensitivity Index (ASI)

Bem Sex Role Inventory (BSRI)

Beliefs of negative implications of anxiety experiences, 18 items [82].

Subjective gender roles, 30 items [83, 84].

Body Awareness Questionnaire

Connor-Davidson Resilience

Scale (CD-RISC)

Dimensional Anhedonia Rating

Scale (DARS)

Emotion Regulation

Questionnaire (ERQ)

Green Space Questionnaire

(GSQ) and geographic info

Maltreatment and Abuse Chronology of Exposure (MACE)

Perceived Social Status Scale (PSS-S)

Ruminative Thought Style Questionnaire (RTS)

Sensitivity to Punishment and

Sensitivity to Reward

Questionnaire (SPSRQ)
Subjective attentiveness to non-emotive body processes, such as the sensitivity to body cycles and rhythms, the ability to detect small changes in normal functioning, and the ability to anticipate bodily reactions, 18 items [85].

Subjective ability to cope with stress, 10 items [86].

Multiple facets of hedonic function such as desire, motivation, effort, and consummatory pleasure across hedonic domains, 17 items [87]

Habitual use of the emotion regulation strategies cognitive reappraisal and expressive suppression, 10-items [88]

Self-developed questionnaire assessing the degree to which participants have access to and make use of green spaces (parks, forests) in their living environment. In combination, geographic analysis of a participant's address data is employed to determine the degree of green space in their living environment, 12 items.

Abuse and neglect during development, 52 items [89].

Subjective socioeconomic status by means of a drawing of a ladder with 10 rungs, described to represent where people stand in society. Participants are instructed to indicate the rung that best represents where they stand on the ladder. Additionally, the same question is asked for the dimensions of academic and occupational status [90].

Components of ruminative thinking including problem-focused thoughts, counterfactual thinking, repetitive thoughts, and anticipatory thoughts, 15 items [91].

Tendency for aversive and appetitive behavior, 48 items [92]. 
State Trait Anxiety Inventory

Symptoms of anxiety as a state and as a general trait, respectively, 40 items [93].

(STAI)

Toronto Alexithymia Scale (TAS- Deficiency in understanding, processing, or describing emotions, 20 items [94]. 20)

Table 4. List of self-report questionnaires. Please note that only the original publications are cited here but not the validation studies of translated versions into the four study languages. Self-developed questionnaires are provided at https://osf.io/qj9mf/. 